\title{
ERGONOMÍA DEL TRABAJO AGRÍCOLA EN LADERAS DE CERRO EN LA ZONA CENTRAL DE CHILE: ESTUDIO DE CASO EN FAENAS DE RIEGO
}

\author{
ERGONOMICS IN HILLSIDE AGRICULTURAL WORK IN CENTRAL ZONE OF CHILE: A \\ CASE STUDY OF IRRIGATION JOB
}

\author{
Daniel Patricio Zavala Briceño ${ }^{1}$
}

\begin{abstract}
Resumen: El trabajo agrícola de producción de frutas constituye una actividad económica de relevancia en la zona central de Chile. En muchos predios se han implementado sistemas de cultivo aprovechando las laderas de los cerros, por lo que los trabajadores han debido adaptarse a efectuar las tareas agrícolas en terrenos con pendiente de variable magnitud y expuestos a altas temperaturas. Se ha diseñado un estudio destinado a conocer las características del trabajo agrícola de frutales en laderas de cerro y su impacto en los trabajadores dedicados a revisión de riego. Se efectúan evaluaciones de carga física mediante la determinación de la carga cardiovascular de los trabajadores, además se obtiene información acerca de su alimentación cotidiana y de los hábitos de hidratación durante el trabajo. También se recaba información ambiental a través de la medición de temperatura seca y humedad relativa en los sitios de trabajo. Se trata de una faena que demanda niveles moderados a altos de carga cardiovascular, que requiere buena aptitud física por parte de quienes la ejecutan, con niveles altos de exposición a calor en los meses estivales. La actividad es más intensa por las tardes debido a la mayor duración de la jornada y al aumento de temperatura ambiental. Conlleva riesgos relacionados al tipo de terreno y su pendiente, como caídas que ocasionan lesiones del sistema musculoesquelético. Se proponen medidas de mitigación del impacto que produce en los trabajadores, tanto personales como organizacionales, orientadas a mejorar las condiciones de desempeño de las tareas agrícolas de laderas.
\end{abstract}

Palabras clave: Ergonomía, agricultura, cultivo en laderas.

\begin{abstract}
The agricultural work of fruit production constitutes an economic activity of relevance in the central zone of Chile. In many farms, cultivation systems have been implemented taking advantage of the slopes of the hills, for which the workers have had to adapt to carry out agricultural tasks on lands with slopes of variable magnitude and exposed to high temperatures. A study has been designed to determine the characteristics of the agricultural work on hillsides and its impact on workers dedicated to irrigation review. Physical load evaluations are carried out by determining the cardiovascular load of the workers, in addition to obtaining information about their daily diet and hydration habits during work. Environmental information was also collected through the measurement of dry temperature and relative humidity at work sites. In summary, it is a task that demands moderate to high levels of cardiovascular load, which requires good physical fitness of those who perform it, with high levels of exposure to heat in summer months. The activity is more intense in the afternoons due to the longer duration of the day and the increase in environmental temperature. It involves risks related to the type of terrain, such as falls that can cause various injuries to
\end{abstract}

${ }^{1}$ Facultad de Medicina Universidad de Chile. Santiago, Chile. Correo electrónico: dzavala@med.uchile.cl. Orcid: https://orcid.org/oooo-0oo1-9381-179o 
musculoskeletal system. Finally, various measures are proposed to mitigate the impact it produces on workers, both personal and organizational, aimed at improving the performance conditions of hillside agricultural tasks.

Keywords: Ergonomics, agriculture, hillside farming.

Recepción: 21.09.2020 / Revisión: 12.10.2020 / Aceptación: 04.12.2020

\section{Introducción}

\section{Ergonomía y agricultura}

En la zona central de Chile, la Región Metropolitana y la V Región concentran buena parte de la producción de frutas en el país. El proceso productivo se exhibe completo en esta área, desde la plantación en invernaderos hasta el packing para su comercialización en el exterior o en el mercado interno. Una rápida vista a los predios que cultivan frutas pone de manifiesto diversos aspectos: primero, que son una fuente muy importante de mano de obra, especialmente para las comunidades rurales que las circundan; segundo, que junto con lo anterior constituyen también con frecuencia fuentes de trabajo precario, sobre todo temporal, en el cual los trabajadores no cuentan con elementos de protección personal, entrenamiento en técnicas de ejecución del trabajo, ni con herramientas adecuadas para ello; y tercero, por último un análisis un poco más profundo revela la importante incongruencia que existe entre las características propias del trabajador o trabajadora, y las demandas del trabajo. No hay estudios antropométricos del trabajador rural de la fruta, que orienten acerca de cómo debería enfocarse el diseño o rediseño de herramientas de mano, de mesones de selección de fruta, de alturas de la poda en parronales, no hay investigación acerca de la fuerza muscular voluntaria de quienes trabajan en cosecha de frutos en altura, en poda de viñedos, o transportando cajas de fruta de exportación. La mayor parte de las maquinarias se instalan y funcionan asumiendo que el trabajador debe adecuarse a ellas. Es claro que no existe investigación suficiente que sustente la adopción de normas y criterios productivos centrados en las características de quién ejecuta el trabajo, y es probable que el aporte que la ergonomía pueda ofrecer signifique mejoras sustantivas en ese sentido. Lo cierto es que esto no parece ser un problema exclusivo de Chile: Schuman, en 2002, efectúa una revisión en Medline entre los años 1996 y 2000, y solo obtiene 11 artículos relacionados a agricultura y ergonomía, y enfatiza la importancia de relevar el estudio de las condiciones en que millones de personas trabajan cada día. Dado el inmenso impacto que tiene la agricultura en la economía y en la supervivencia de las poblaciones de muchos países, especialmente pobres o en vías de desarrollo, la investigación de las características propias de los sistemas productivos cobra mayor relevancia. Según datos de la OIT, en América Latina el año 2006, 31 millones de personas tenían empleo en la agricultura, en tanto que los asalariados en el mismo sector alcanzarían los 100 millones, de los cuales un 30\% son mujeres (Organización Internacional del Trabajo [ILO] , 2014, 16 de enero).

En la búsqueda de literatura relativa a ergonomía y agricultura, destacan gran cantidad de trabajos efectuados en la India, estudios de antropometría (Dewangan et al., 2008; Dewangan, Owary \& Datta, 2010; Chakrabarti, \& Bhattachheriya, 2012) de hombres y 
mujeres agricultores de sobrevivencia para el diseño de herramientas manuales menos riesgosas, técnicas para controlar el trabajo pesado en mujeres (Kishtwaria \& Rana, 2012; Mehta et al., 2008), mediciones de fuerza muscular voluntaria en hombres y mujeres (Dewangan, Gogoi, Owary \& Gorate, 2010; Agrawal et al., 2009). Otro aspecto se refiere a la determinación de fuerza voluntaria y antropometría para el diseño de tractores (Tiwari et al., 2010; Mehta et al., 2008), evaluación ergonómica del uso de plataformas mecánicas para la cosecha de frutas en altura (Alves-Costa \& Camarotto, 2012). La determinación de variables térmicas en el trabajo en invernaderos (Callejon-Ferre et al., 2011); y numerosos trabajos relativos a los TME (trastornos musculoesqueléticos) en faenas agrícolas (Anderson et al., 2007; Jiang et al., 2005; Earle-Richardson et al., 2005; Kirkhorn et al., 2010; Innes \& Walsh, 2010).

Respecto al tema de la agricultura en laderas, se registra un trabajo efectuado en India referente a la carga de trabajo de mujeres en labores de desmalezado manual en cultivos de laderas (Kishtwaria \& Rana, 2012). A propósito de los temas ambientales, se evalúa la validez, ambigüedad y facilidad de uso de diferentes estándares de medición de carga térmica en países en vías de desarrollo, y puntualmente en labores agrícolas (McNeill \& Parsons, 1999). Otro estudio valora el desempeño de cosechadores de café en el trópico (Bao et al., 2013). En Chile no se registran trabajos relacionados al tema.

\section{Cultivos de frutales en laderas}

En los últimos 15 años se ha introducido en Chile, específicamente en la zona central, una modalidad de cultivo de frutales que se ha masificado rápidamente debido a ciertas ventajas productivas respecto a los métodos tradicionales (Ruiz, 2005).

Este avance en las técnicas agrícolas ha traído aparejado que una gran cantidad de predios hayan trasladado sus huertos, fundamentalmente de paltas y cítricos, a los cerros. De acuerdo a datos proporcionados por el Censo Agropecuario y Forestal (Instituto Nacional de Estadística de Chile [INE-Chile], 2007), el país cuenta con 30.891há dedicadas al cultivo de paltas, en sus diversas variedades, el $75 \%$ de las cuales se halla entre las regiones $\mathrm{V}$ y Metropolitana. En la Provincia de Melipilla, en tanto, se encuentra el 65\% de las plantaciones de este fruto en la RM. Respecto de los cítricos, puntualmente naranjas y mandarinos, las plantaciones ascienden a 7.378há en el país, $27,5 \%$ en la RM, y el 80\% de estas en Melipilla. Es posible suponer entonces, debido a que no hay una cuantificación oficial de la cantidad de cultivos en ladera en la zona central hasta el momento, que buena parte de los predios destinados a la producción de estos frutos lo están haciendo incorporando técnicas de ladera y aprovechando estos terrenos en sus huertos. En consecuencia, a ello se han debido sumar los trabajadores agrícolas, efectuando esencialmente las mismas tareas de plantación, regadío, cosecha, y otras, pero en un terreno que demanda necesariamente niveles diferentes de desempeño y esfuerzo.

En este nuevo escenario, los trabajadores están expuestos a condiciones ambientales diferentes a las que se verifican en terrenos planos. Muchas tareas demandan subir y bajar frecuentemente por laderas de diversa pendiente y longitud; el nivel de exposición solar, la temperatura ambiental en el cerro y la consiguiente deshidratación en períodos de primavera 
o verano, cuando aumenta la actividad en el huerto, son elementos que pueden afectar la capacidad de desempeño de los trabajadores.

El tema de investigación del presente estudio se refiere, entonces, a la determinación de las características del trabajo agrícola en laderas de cerro, y la cuantificación de los niveles de carga física y ambiental a que se ven expuestos los trabajadores agrícolas de laderas en los meses de primavera y verano. A esto, se suma el estudio de variables relacionadas a la hidratación y al balance nutricional de los trabajadores expuestos, como una medida para evaluar su calidad de vida laboral en el ejercicio de sus puestos de trabajo.

\section{Objetivos}

1. Determinar las características del trabajo agrícola en laderas de cerro en la zona central de Chile.

2. Evaluar la carga física a la que están expuestos los trabajadores agrícolas de ladera.

3. Conocer las condiciones ambientales en que se desarrolla el trabajo en laderas.

4. Evaluar las condiciones de hidratación y alimentación de los trabajadores de ladera.

5. Determinar las características organizacionales de una unidad productiva agrícola con cultivos en laderas.

6. Proponer medidas para la mejora de condiciones de sobrecarga de trabajo.

\section{Materiales y métodos}

\section{Empresa}

El presente trabajo se desarrolló íntegramente en el predio perteneciente al de una sociedad agrícola ubicado en la Provincia de Melipilla, Región Metropolitana de Chile, en los meses de noviembre y diciembre de 2013. La unidad productiva cuenta con un terreno de 63ohá totales correspondiente al tipo de secano montañoso. Las tareas agrícolas que se desarrollan en la unidad pueden ser resumidas como sigue:

- Riego.

- Aplicaciones (abonos, pesticidas, etc.).

- Cosecha.

- Aplicación manual de herbicidas.

- Desmalezado manual.

- Poda.

- Instalación de riego.

- Plantación.

La administración señala que el puesto de trabajo más expuesto a la faena en laderas es el de riego (o encargados de revisión de riego), que básicamente consiste en recorrer los cultivos, divididos por sectores, mientras se procede a su riego, con el fin de verificar que 
cada planta está recibiendo la cantidad adecuada de agua. En el tiempo en que se efectuó el presente estudio, la faena de revisión de riego fue la única actividad permanente en todos los sectores del predio. Esto, sumado a que coincide con los períodos de mayor temperatura, permitió focalizar la investigación de carga de trabajo y ambiental en esa labor. Para la recolección de datos se consideró la participación de dos trabajadores permanentes en esta faena. En la tabla 1 se resumen sus características.

Tabla 1. Datos de trabajadores evaluados.

\begin{tabular}{|l|c|c|}
\hline & Trabajador 1 (W1) & Trabajador 2 (W2) \\
\hline Edad & 21 años & 47 años \\
\hline Talla & $166 \mathrm{~cm}$ & $165 \mathrm{~cm}$ \\
\hline Peso & $65 \mathrm{~kg}$ & $58 \mathrm{~kg}$ \\
\hline IMC & 23,6 & 21,3 \\
\hline Permanencia & 3 años & 8 años \\
\hline
\end{tabular}

\section{Instrumentación utilizada}

- Psicrómetro de voleo.

- Termómetro ambiental digital.

- Cinta métrica - regla metálica.

- Balanza común.

- Polar RS80o con altímetro y GPS.

- Pulsómetros Polar RS40o.

- Cámara fotográfica Sony DS-W35.

\section{Metodología}

La determinación de la carga física se efectúo utilizando pulsómetros Polar, modelos RS40o y RS80o, los que se colocaban en los trabajadores destinados a revisión de riego al comenzar la jornada, y se retiraban por la tarde al finalizarla, totalizando 10 horas y media de registro por día. El mismo equipo proporcionó los datos de altitud sobre el nivel del mar, necesarios para determinar los ascensos y descensos durante el trabajo. Además, y gracias a la incorporación de un GPS, se obtuvo el registro de la longitud de los recorridos, y la cantidad de tiempo invertida en subidas y bajadas, así como una imagen satelital del terreno cubierto durante la jornada. Es necesario reseñar que los trabajadores de riego están en pendiente la mayor parte de su tiempo de trabajo.

Respecto de la evaluación ambiental, se utilizó un psicrómetro de manivela o voleo, con el cual se determinó la temperatura del aire (Tbs) y la de bulbo húmedo, registro que se efectuó cada hora siguiendo el recorrido de los trabajadores. Además, se utilizó un termómetro ambiental digital con varilla, que permitió tener un valor alternativo respecto de la temperatura del aire. 
En lo referente a la alimentación, se aplicó el método recordatorio 24 horas a algunos trabajadores del predio, mediante una encuesta simple, en la cual se consultó a los trabajadores presentes en el predio acerca de los contenidos habituales de sus comidas, considerando desayuno, almuerzo y cena.

La hidratación diaria durante las horas de trabajo también fue un tema en que se consultó a los trabajadores, y se determinó una cantidad promedio de ingesta de líquido antes, durante y después de la jornada.

\section{Resultados y discusión}

\section{Carga de trabajo}

Las mediciones de carga de trabajo, como fuera mencionado, se efectuaron midiendo la FC (frecuencia cardíaca) de los trabajadores con pulsómetros Polar, los cuales registraron el ritmo cardíaco cada 5 segundos durante toda la jornada, la que fue dividida en 10 horas. Si bien la utilización de esta variable no permite evaluar directamente el gasto de energía de los trabajadores, sí da una idea fidedigna acerca de los niveles de esfuerzo a que se encuentran sometidos (Apud et al., 2003). En cada caso se procedió a calcular el \%CC (\% de carga cardiovascular) por hora de trabajo y su promedio por jornada. Se trata de dos personas bien aclimatadas y con experiencia de varios años en la tarea.

La medición de FC durante la jornada arrojó información acerca de los niveles de \%CC de ambos trabajadores. En la tabla 2 se resumen los datos obtenidos.

Respecto de los datos obtenidos de FC, es claro que la determinación del \%CC diario no excede los límites peligrosos de carga (Apud et al., 2002), aun cuando el promedio de FC de la jornada de W1 (trabajador 1) fue de 97,8 latidos/min y el de W2 (trabajador 2) alcanzó los 99 latidos/min.

Tabla 2. Promedios de FC por hora y \%CC en ambos trabajadores.

\begin{tabular}{|c|c|c|c|c|}
\hline & \multicolumn{2}{|c|}{ W1 } & \multicolumn{2}{c|}{ W2 } \\
\hline Tiempo & X FC & \%CC & X FC & \%CC \\
\hline H1 & 103,2 & 31,1 & 108,9 & 41,0 \\
\hline H2 & 105,5 & 32,7 & 106,8 & 39,1 \\
\hline H3 & 98,1 & 27,4 & 101,1 & 33,7 \\
\hline H4 & 92,3 & 23,2 & 98,1 & 30,9 \\
\hline H5 & 90,6 & 22,0 & 89,9 & 23,3 \\
\hline H6 & 90,9 & 22,2 & 102,8 & 35,3 \\
\hline H7 & 89,4 & 21,2 & 99,6 & 32,3 \\
\hline H8 & 95,6 & 25,6 & 92,9 & 26,1 \\
\hline H9 & 103,3 & 31,2 & 94 & 27,1 \\
\hline H10 & 107,9 & 34,5 & 94,9 & 27,9 \\
\hline H11 & & & 100,4 & 33,1 \\
\hline \%CC diario & & 27,1 & & 31,8 \\
\hline
\end{tabular}


En ambos casos, además, se alcanzaron FC máximas de 166 lat/min en W1, y de 136 lat/min en W2. A modo de ejemplo, se aprecian las curvas diarias de FC y altitud en un trabajador en una gráfica proporcionada por el sistema Polar (figura 1). En esta gráfica es visible la necesidad de efectuar frecuentes subidas y bajadas por el terreno. De una altura base de 260msnm, los trabajadores frecuentemente suben hasta más de 300msnm, incluso $380 m s n m$ dependiendo de las zonas a cubrir. Se aprecia también que los ascensos bruscos de FC se dan tanto en las subidas como en las bajadas. Con alguna frecuencia los trabajadores moderan su actividad en lo que parecen pausas no programadas, y en las cuales logran FC de menos de 80 lat/min. En esto se evidencia una tendencia natural a la autorregulación, seguramente practicada en forma permanente, como una forma de cumplir con la faena encargada.

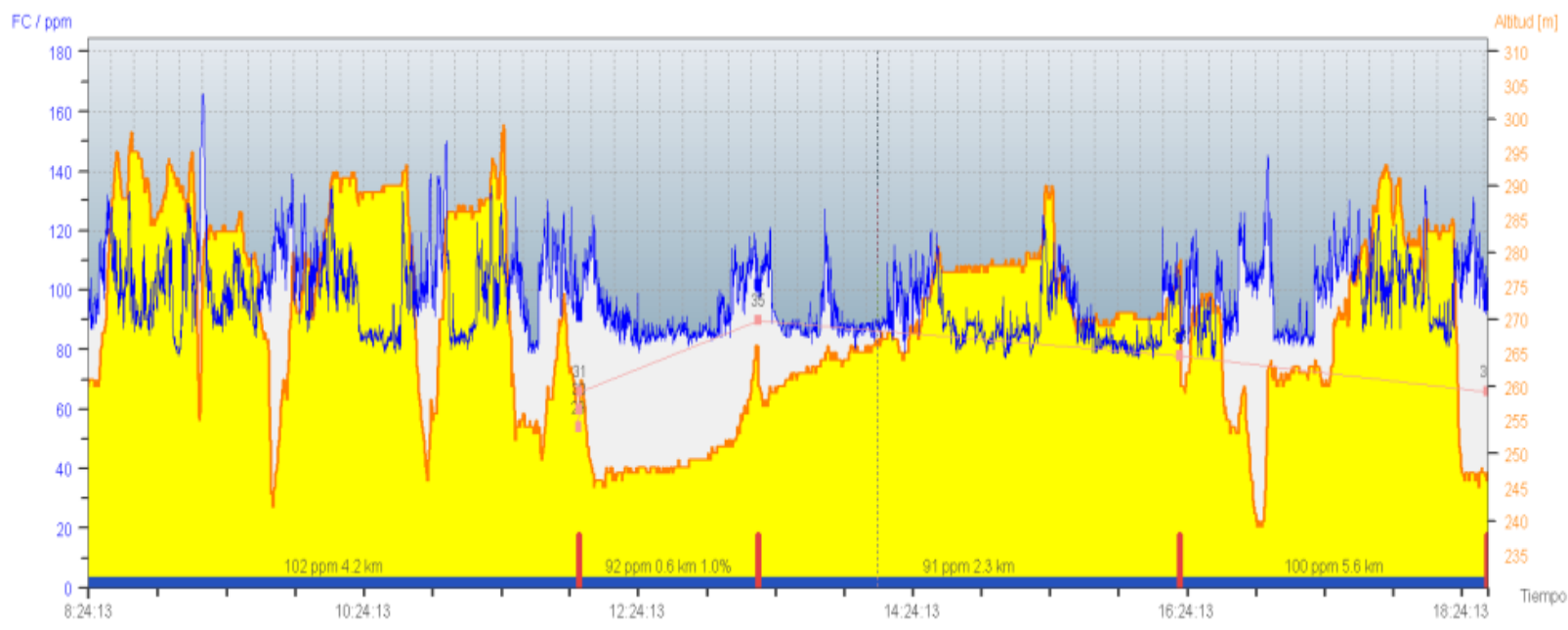

Figura 1. Curvas de FC (azul) y altitud (amarillo) durante la jornada de trabajo.

La utilización de un GPS permitió conocer en detalle los recorridos efectuados por los trabajadores, los cuales normalmente pueden recorrer $13 \mathrm{~km}$ diarios durante la faena.

También se obtuvo que en general el recorrido de la tarde es más corto que el de la mañana, a pesar de ser esta jornada más larga, posiblemente por un mecanismo de regulación que podría estar relacionado a la mayor temperatura. También las pausas no programadas son más frecuentes durante la tarde.

Respecto de las alturas, la tabla 3 contiene datos acerca de los ascensos por hora. Si se efectúa una relación entre los metros de ascenso contemplados en la tabla 3 y los valores de \%CC obtenidos para la jornada por hora, es posible conseguir un valor que se acerque a un rendimiento o costo de la actividad, como se observa en la tabla 4 (Apud et al., 2002). 
Tabla 3. Ascensos por hora de trabajo y diferencias de altitud, en msnm.

\begin{tabular}{|c|c|c|c|}
\hline Hora & Min & Max & Dif \\
\hline H1 & 260 & 361 & 101 \\
\hline H2 & 335 & 380 & 45 \\
\hline H3 & 342 & 380 & 38 \\
\hline H4 & 255 & 363 & 108 \\
\hline H5 pausa & 255 & 259 & 4 \\
\hline H6 & 256 & 316 & 60 \\
\hline H7 & 316 & 345 & 29 \\
\hline H8 & 305 & 341 & 36 \\
\hline H9 & 307 & 351 & 44 \\
\hline H10 & 322 & 373 & 51 \\
\hline H11 & 263 & 321 & 58 \\
\hline & & Promedio: & 52,2 \\
\hline
\end{tabular}

Otro método que propone una determinación del gasto energético usando la frecuencia cardíaca y la velocidad de desplazamiento, el PCI (Physiological Cost Index), utilizado por diversos aparatos de entrenamiento, no consigue niveles de confiabilidad adecuados en pruebas de laboratorio de correlación con métodos tradicionales (Graham et al., 2005). En otro caso, el TEE (Total Energy Expenditure), basado en el software MoveSense HRAnalyzer 2011, desarrollado por la empresa finlandesa Suunto, consigue mejores grados de respuesta, pero requiere ser probado en situaciones diferentes a las de laboratorio para asegurar su confiabilidad y validez (Yu et al., 2012). Hood et al. (2002) propone en ese sentido el uso del THBI, indicador basado en la cantidad total de latidos y la distancia recorrida, muy usado en procedimientos de rehabilitación de marcha. Smolander et al. (2008) evalúa el uso de otro software, el FirstBeat PRO VO2-HRV, para el cálculo del consumo de oxígeno en terreno en individuos sin calibración previa.

Los datos de los termómetros de bulbo seco (BS) y bulbo húmedo (BH) permitieron utilizar una tabla de cálculo de humedad relativa (HR) (Mondelo et al., 2001). De acuerdo a lo observado, las horas de la tarde son claramente más calurosas que las de la mañana, y generalmente el trabajo se efectuó en sectores en los cuales la sombra provista por los árboles fue más bien escasa. 
Tabla 4. Promedio por jornada de mañana y tarde en relación al \%CC y metros ascendidos.

\begin{tabular}{|c|c|c|c|c|c|}
\hline Hora & \%CC & m ascenso & $\% \mathrm{CC} / \mathrm{m}$ & X Jornada & Jornada \\
\hline $\mathrm{H} 1$ & 41,03 & 101 & 0,41 & \multirow{4}{*}{0,61} & \multirow{4}{*}{ mañana } \\
\hline $\mathrm{H} 2$ & 39,07 & 45 & 0,87 & & \\
\hline $\mathrm{H}_{3}$ & 33,74 & 38 & 0,89 & & \\
\hline $\mathrm{H} 4$ & 30,93 & 108 & 0,29 & & \\
\hline $\mathrm{H} 5$ & \multicolumn{5}{|c|}{ colación } \\
\hline H6 & 35,33 & 60 & 0,59 & \multirow{6}{*}{0,69} & \multirow{6}{*}{ tarde } \\
\hline $\mathrm{H}_{7}$ & 32,34 & 29 & 1,12 & & \\
\hline H8 & 26,07 & 36 & 0,72 & & \\
\hline H9 & 27,10 & 44 & 0,62 & & \\
\hline H10 & 27,94 & 51 & 0,55 & & \\
\hline H11 & 33,08 & 58 & 0,57 & & \\
\hline
\end{tabular}

La relación de \%CC/m de ascenso permite observar un mayor coeficiente por las tardes, lo que indicaría un mayor esfuerzo por metro ascendido en dichas jornadas.

\section{Carga térmica}

Durante el trabajo de campo, se obtuvieron datos de temperatura y humedad ambiental en el terreno, para lo cual las mediciones fueron hechas cerca de los lugares donde los trabajadores efectuaban sus faenas. En general, se contó con días soleados, sin nubosidad, y con presencia permanente de viento por las tardes, de entre 0,5 y $1 \mathrm{~m} / \mathrm{s}$ (brisa) (Mondelo et al., 2001), el que aumentaba ligeramente después de las 16 horas. Las temperaturas, en tanto, fluctuaban entre los 15 y $19^{\circ} \mathrm{C}$ la mínima, y entre 30 y $35^{\circ} \mathrm{C}$ la máxima.

En la medición se utilizó la temperatura de BS y BH, además de un termómetro digital de uso regular en el predio (TD). La tabla 5 resume los datos de temperatura recogidos durante una jornada de trabajo.

Tabla 5. Temperaturas de BS, BH, TD y determinación de HR.

\begin{tabular}{|c|c|c|c|c|}
\hline & \multicolumn{3}{|c|}{$\mathbf{T}^{\mathbf{2}}$} & \\
\hline Hora & BS & BH & TD & HR \\
\hline H1 & 19 & 16 & 25,3 & 72 \\
\hline H2 & 24 & 16 & 28,1 & 42 \\
\hline H3 & 28,5 & 18 & 30,5 & 37 \\
\hline H4 & 30,5 & 20 & 32,5 & 40 \\
\hline H5 & 31,4 & 21 & 33 & 41 \\
\hline H6 & 33 & 22 & 34,5 & 38 \\
\hline H7 & 35 & 23 & 35,2 & 36 \\
\hline H8 & 28 & 21 & 31,5 & 53 \\
\hline H9 & 29 & 23 & 32,2 & 60 \\
\hline H10 & 28 & 24 & 31,4 & 72 \\
\hline
\end{tabular}


Haciendo una asociación entre el \%CC y el promedio de las temperaturas secas (BS y TD), se obtiene el gráfico de la figura 2. Se aprecia cómo la CC del W2 se inicia muy alta al comienzo de la mañana para llegar a su punto más bajo de esa jornada con la pausa del almuerzo. Luego sube nuevamente al comenzar la jornada de la tarde, seguramente debido al traslado hasta el punto de trabajo, para descender y tender a estabilizarse en el resto de la jornada, lo que coincide con la fluctuación de la temperatura del aire pasado el mediodía. Esto tiene relación con lo observado por Tiwari \& Gite (2006), en su estudio de esquemas de pausa en trabajadores agrícolas hindúes. En el caso del W1, también inicia la jornada con una carga en ascenso, para ir disminuyendo paulatinamente durante el día hasta la hora 7 , en que aumenta hasta alcanzar valores semejantes a los de la mañana.

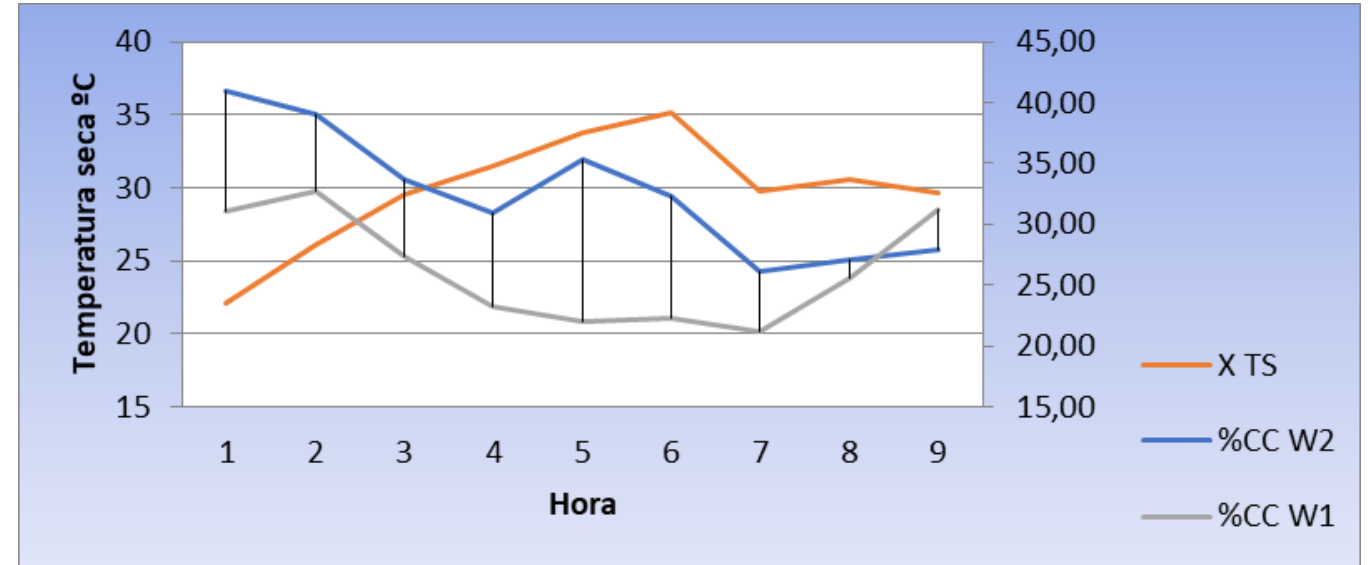

Figura 2. \%CC y temperatura seca, por hora de trabajo (se excluye la hora de colación).

\section{Alimentación}

Todos los trabajadores traen su almuerzo desde sus casas, y la empresa no provee ningún tipo de alimentación extra.

\section{Cálculo de ingesta diaria en Kcal}

Según el análisis efectuado a partir de la encuesta alimenticia aplicada, los trabajadores consumirían diariamente un mínimo de 2786 Kcal hasta un máximo de 3274,5 Kcal. El promedio asciende a 3006,7 Kcal/día. Si bien no se efectuó una evaluación del estado nutricional y del porcentaje de masa grasa de los trabajadores, la percepción es que la mayoría corresponden al tipo mesomorfo y ectomorfo.

Respecto de la energía consumida en el trabajo, la FC por sí sola no permite su determinación con niveles de exactitud aceptables. Al usar alguna de las tablas de estimación del metabolismo disponibles en la literatura , en este caso la de Lehmann (1960), se obtiene un nivel de gasto energético de sobre $5000 \mathrm{Kcal} /$ día, lo cual lo sitúa muy por encima de la ingesta que se ha calculado, en cuyo caso existiría un marcado desbalance energético (Mondelo et al., 2001). 


\section{Hidratación}

La cuantificación del consumo de líquidos por parte de los trabajadores de riego se efectuó mediante un cuestionario y la observación directa de los hábitos en la faena. En general, en la jornada de mañana no consumen agua ni la portan en sus morrales.

Por la tarde, el trabajador de más edad (W2) porta una botella de 1,5 o 2 litros de agua, la que consume completamente durante la jornada. Al volver del cerro, ambos toman directamente de la llave una cantidad indeterminada de agua, y según su percepción con mucha sed.

En el caso del trabajador más joven (W1), frecuentemente no lleva agua en la tarde. En esto coincide con lo observado por Apud et al. (2002), en relación a los hábitos de ingesta de líquidos en los brigadistas forestales. En este sentido, también se analizan las CC de ambos trabajadores por la tarde (figura 3).

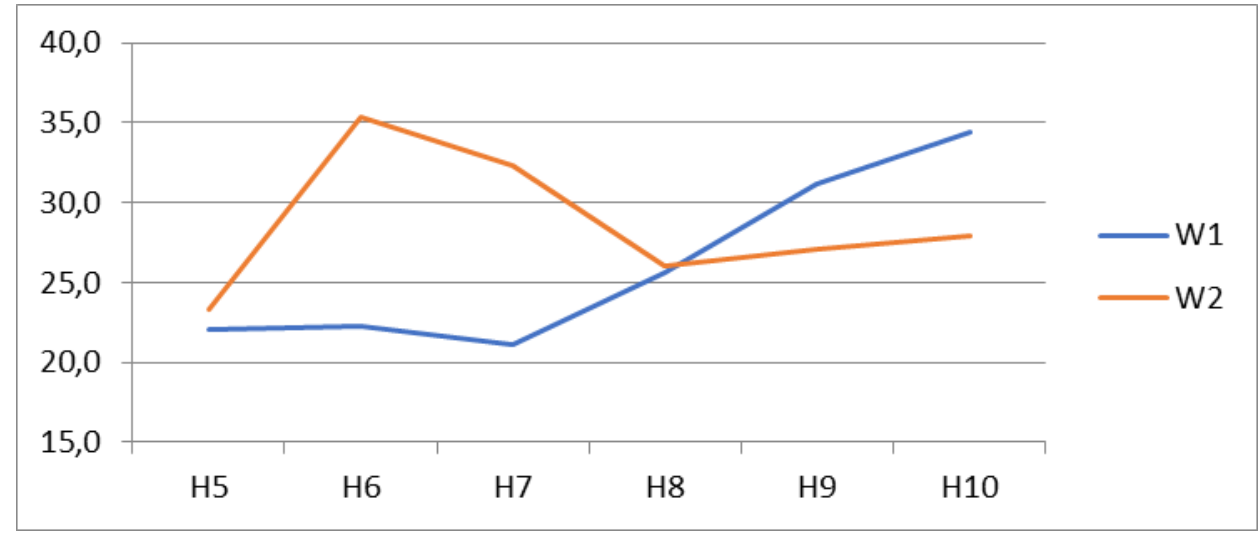

Figura 3. \%CC de trabajadores en horas de jornada de tarde.

En el análisis del gráfico de la figura 3, se nota un incremento gradual de la CC del trabajador más joven que no consume agua durante la tarde, llegando a un máximo de $35 \%$ al terminar la jornada, lo que podría revelar un aumento en su gasto cardíaco secundario al estrés térmico. En el caso de W2, el trabajador de más edad, revela un ascenso inicial de su CC, para después descender y estabilizarse en valores cercanos a 25\%. Esto pudiera relacionarse en parte a su hábito de ingesta gradual de agua durante la jornada (Apud et al., 2003).

\section{Vestimenta}

Los trabajadores usan vestuario provisto por la empresa, además de ropa propia bajo el buzo o sobre él. Por las tardes, frecuentemente se quitan la parte superior del buzo, quedando vestidos con una polera de algodón. El buzo, de popelina o material similar, no es caluroso, de acuerdo a lo que manifiestan, pero cuando la temperatura sube no permite la salida de sudor ni la convección, al no tener en su diseño aberturas que la faciliten. 


\section{Conclusiones}

En la medición de la FC de los trabajadores se obtuvieron datos acerca de la carga a que están sometidos durante la faena. Sin duda, se trata de un trabajo que demanda esfuerzos importantes y permanentes durante la jornada, que si bien no están expuestos a niveles peligrosos de carga, ni sobrepasan en forma frecuente el límite de 40\% de su CC, que está en la base de una calificación de trabajo pesado (Apud et al., 2002), pueden alcanzar máximos de FC riesgosos para su salud. Respecto a la distribución de su jornada, y a la necesidad de pausas, se propone un cálculo de tiempos de recuperación basado en las horas de trabajo.

La asociación entre la CC y el diferencial de altitud, que se utiliza como una medida de rendimiento, permite conocer el costo del trabajo, y determinar objetivamente que este es mayor por las tardes, jornada más prolongada en una hora y en que la temperatura es mayor. La tecnología GPS también entrega datos que refrendan lo anterior: el recorrido de la tarde es $2 \mathrm{~km}$ más corto.

Respecto del ambiente térmico, se constató un aumento sostenido de la temperatura del aire hasta las 16 horas aproximadamente, lo que redunda en el rendimiento de ambos trabajadores. Posiblemente estos permanecen buena parte de la jornada de la tarde expuestos a la radiación solar. Si esto es asociado al factor hidratación, es de notar cómo el W2 logra mantener relativamente estables sus niveles de CC, debido a que ingiere líquido mientras está en faena; el W1, que habitualmente no se hidrata, experimenta un ascenso de casi un $15 \%$ en las últimas horas de trabajo. Esto claramente puede tener relación directa con la deshidratación a que se ve expuesto.

La alimentación, de acuerdo al análisis efectuado, provee alrededor de 3000 Kcal por día. El trabajo de revisión de riego, en tanto, puede significar un gasto mayor, si objetivamente se considera el \%CC de cada persona, los trayectos que efectúan a diario, las pendientes en que trabajan y la temperatura ambiental. De acuerdo a la literatura disponible, el trabajo ejecutado podría situarse entre moderado y pesado de acuerdo a su valor de costo energético promedio, esto es, entre 375 y $450 \mathrm{Kcal} / \mathrm{h}$, lo cual arrojaría por jornada efectiva de 9 horas 30 minutos, un gasto de entre 3563 y 4275 Kcal. Probable es entonces que este gasto energético mayor se refleje en la delgadez de los trabajadores. Sin embargo, en ninguno de los dos casos hay reportes de falta de resistencia física para ejecutar sus labores, y de hecho son seleccionados para ello por su capacidad.

En cuanto a la hidratación, la cantidad de líquido ingerida por los trabajadores es claramente insuficiente para los requerimientos de la faena, al menos en el período primavera-verano. Un indicador es la sed con que vuelven habitualmente del cerro. En esta oportunidad no se evaluó el nivel de pérdida de líquidos en los trabajadores, lo cual daría una medida objetiva acerca de cuánto es necesario proveerles para hidratarlos convenientemente.

Finalmente, la información recabada y analizada respecto a las características del puesto permite resumir las siguientes conclusiones:

- Se trata de una labor que demanda aptitud física por parte de quienes la ejercen, con un alto nivel de exposición a esfuerzo y tolerancia a condiciones climáticas de alta temperatura. 
- El puesto se desarrolla fundamentalmente en laderas de diverso grado de pendiente.

- La jornada de la tarde es más demandante que la de mañana, al ser más prolongada y con mayor exposición a calor.

- Los trabajadores, de manera informal, organizan su propio sistema de pausas, como una medida de autorregulación. La empresa no prevé ningún sistema de pausas durante la jornada, excepto la de mediodía para colación.

- No existen en la actualidad sistemas de provisión de agua fresca en terreno.

\section{Referencias}

Agrawal, K., Singh, R., \& Satapathy, K. (2009). Isometric strength of agricultural workers of Meghalaya: A case study of an Indian population, International Journal of Industrial Ergonomics, 39 (6) 919-923. http://dx.doi.org/10.1016/j.ergon.2009.06.008

Alves-Costa S., \& Camarotto, J. (2012). An ergonomics approach to citrus harvest mechanization. Work, 41(1), 5027-5032. https://doi.org/10.3233/WOR-2012-0794-5027

Anderson, A.M., Meador, K.A., McClure, L.R., Makrozahopoulos, D., Brooks, D.J., \& Mirka, G.A. (2007). A biomechanical analysis of anterior load carriage. Ergonomics, 5O(12), 2104-2117. https://doi.org/10.1080/00140130701450195

Apud, E., Gutiérrez, M., Maureira, F., Lagos, S., Meyer, F. \& Chiang, M.T. (2003). Guía para la evaluación de trabajos pesados. Universidad de Concepción.

Apud, E., Meyer, F., Maureira, F., Lagos, S., Espinoza, J. \& Lecanelier, E., (2002). Ergonomía en el combate de incendios forestales. Universidad de Concepción.

Bao, S., Silverstein, B., \& Stewart, K. (2013). Evaluation of an ergonomics intervention among Nicaraguan coffee harvesting workers. Ergonomics, 56(2), 166-181. http://doi.org/10.1080/00140139.2012.760753

Callejon-Ferre A., Manzano-Agugliaro F., Díaz-Pérez, M., \& Carreno-Sánchez, J. (2011). Improving the climate safety of workers in Almería-type greenhouses in Spain by predicting the periods when they are most likely to suffer thermal stress. Applied Ergonomics, 42(2) 391-396. https://doi.org/10.1016/j.apergo.2010.08.014

Chakrabarti, D., \& Bhattachheriya, N. (2012). Ergonomic design intervention strategy for work tools development for women agro based workers in Northeast India. Work, 41(1), 13301334. https://doi.org/10.3233/wor-2012-0319-1330

Dewangan, K.N., Owary, C., \& Datta, K. (2008). Anthropometric data of female farm workers from north eastern India and design of hand tools of the hilly region. International Journal of Industrial Ergonomics, 38(1), 90-100. https://doi.org/10.1016/j.ergon.2007.09.004

Dewangan, K.N., Owary, C., \& Datta, K. (2010). Anthropometry of male agricultural workers of north-eastern India and its use in design of agricultural tools and equipment. International $\begin{array}{llll}\text { Journal of Industrial } & \text { Ergonomics, } & \text { 40(5), }\end{array}$ https://doi.org/10.1016/j.ergon.2010.05.006 
Dewangan K.N., Gogoi, G., Owary, C., \& Gorate, D.U. (2010). Isometric muscle strength of male agricultural workers of India and the design of tractor controls. International Journal of Industrial Ergonomics, 4O(5), 484-491. https://doi.org/10.1016/j.ergon.2010.05.008

Earle-Richardson, G., Jenkins, P., Fulmer, S., Mason, C., Burdick, P., \& May, J. (2005). An ergonomic intervention to reduce back strain among apple harvest workers in New York State. Applied Ergonomics, 36(3), 327-334. https://doi.org/10.1016/j.apergo.2004.12.003

Graham, R.C., Smith, N.M., \&White, C.M. (2005).The reliability and validity of the physiological cost index in healthy subjects while walking on 2 different tracks. Archives of Physical Medicine and Rehabilitation, 86(10), 2041-2046. https://doi.org/10.1016/j.apmr.2005.04.022

Hood, V.L., Granat, M.H., Maxwell, D.J., \& Hasler, J.P. (2002). A new method of using heart rate to represent energy expenditure: The total heart beat index. Archives of Physical Medicine and Rehabilitation, 83(9), 1266-73. https://doi.org/10.1053/apmr.2002.34598

Innes E., \& Walsh C. (2010). Musculoskeletal disorders in Australian dairy farming. Work, 36(2), 141-155. https://doi.org/10.3233/wor-2010-1016

Instituto Nacional de Estadística de Chile (2014, 10 de enero). Censo Agropecuario y Forestal 2007. https://www.ine.cl/estadisticas/economia/agricultura-agroindustria-y-pesca/censosagropecuarios

Jiang, Z., Shin, G., Freeman, J., Reid, S., \& Mirka, G.A. (2005). A study of lifting tasks performed on laterally slanted ground surfaces. Ergonomics, 48(7), $782-795$. https://doi.org/10.1080/00140130500123761

Kirkhorn, S.R., Earle-Richardson, G., \& Banks, R.J. (2010). Ergonomic risks and musculoskeletal disorders in production agriculture: Recommendations for effective research to practice. Journal of Agromedicine, 15(3), 281-299. https://doi.org/10.1080/1059924X.2010.488618

Kishtwaria, J., \& Rana, N. (2012). Ergonomic interventions in weeding operations for drudgery reduction of hill farm women of India. Work, 41(1), 4349-4355. https://doi.org/10.3233/wor-2012-0730-4349

McNeill, M.B., \& Parsons, K.C. (1999). Appropriateness of international heat stress standards for use in tropical agricultural environments. Ergonomics, 42(6), 779-797. https://doi.org/10.1080/001401399185289

Mehta, C.R, Gite, L.P., Pharade, S.C., Majumder, J., \& Pandey, M.M. (2008). Review of anthropometric considerations for tractor seat design. International Journal of Industrial Ergonomics, 38(5-6), 546-554. https://doi.org/10.1016/j.ergon.2007.08.019

Mondelo, P.R., Gregori, T.E., Comas, U.S., Castejón V.E., \& Bartolomé, L.E. (2001). Ergonomía 2: confort y estrés térmico. Ediciones UPC.

Organización Internacional del Trabajo (2014, 16 de enero). ILOSTAT Database. www.ilo.org/ilostat/faces/home/statisticaldata

Ruiz, C.G. (ed.) (2005) Manual de especificaciones técnicas de buenas prácticas de manejo de suelos en laderas. Serie Técnica $\mathrm{N}^{\circ} 20$. Servicio Agrícola y Ganadero, SAG, Santiago de Chile, 56pp. $\quad$ https://es.scribd.com/document/313586014/N\%C2\%BA2O-Manual-de-Buenas- 
Practicas-de-Manejo-de-Suelos-en-Laderas

Schuman, S. (2002) Ergonomics in agriculture. Journal of Agromedicine, 8(1), 9-18. https://doi.org/10.1300/Jog6vo8no1 02

Smolander, J., Juuti, T., Kinnunen, M.L., Laine, K., Louhevaara, V., Männikkö, K., \& Rusko, H. (2008). A new heart rate variability-based method for the estimation of oxygen consumption without individual laboratory calibration: Application example on postal workers. Applied Ergonomics, 39(3) 325-331. https://doi.org/10.1016/j.aperg0.2007.09.001

Tiwari P.S., \& Gite L.P. (2006). Evaluation of work-rest schedules during operation of a rotary power tiller. International Journal of Industrial Ergonomics, 36(3), 203-210. https://doi.org/10.1016/j.ergon.2005.11.001

Tiwari, P.S, Gite, L.P., Majumder, J., Pharade, S.C., \& Singh, V.V. (2010). Push/pull strength of agricultural workers in central India. International Journal of Industrial Ergonomics, 40 (1), 1-7. https://doi.org/10.1016/j.ergon.2009.10.001

Yu, Z., Völgyi, E., Wang, R., Ember, A., Wiklund, P., Alén, M., Tylavsky, F.A., \& Cheng, S. (2012). Comparison of heart rate monitoring with indirect calorimetry for energy expenditure evaluation. Journal of Sport and Health Science, 1(3), 178-183. https://doi.org/10.1016/j.jshs.2012.07.004 